\title{
Are short magnetic resonance imaging protocols the future of prostate imaging?
}

\author{
Massimo Imbriaco, Arnaldo Stanzione, Virginia Tamburi, Michele Gambardella, Andrea Ponsiglione, \\ Renato Cuocolo
}

Department of Advanced Biomedical Sciences, University "Federico II" Napoli, Naples, Italy

Correspondence to: Massimo Imbriaco. Associate Professor of Radiology, Department of Advanced Biomedical Sciences, University "Federico II" Napoli, Naples, Italy. Email: massimo.imbriaco@unina.it.

Provenance and Peer Review: This article was commissioned and reviewed by the Section Editor Xiao Li (Department of Urology, Jiangsu Cancer Hospital \& Jiangsu Institute of Cancer Research \& Nanjing Medical University Af liated Cancer Hospital, Nanjing, China).

Comment on: van der Leest M, Israël B, Cornel EB, et al. High Diagnostic Performance of Short Magnetic Resonance Imaging Protocols for Prostate Cancer Detection in Biopsy-naïve Men: The Next Step in Magnetic Resonance Imaging Accessibility. Eur Urol 2019;76:574-81.

Submitted Aug 30, 2019. Accepted for publication Dec 13, 2019.

doi: $10.21037 /$ cco.2019.12.13

View this article at: http://dx.doi.org/10.21037/cco.2019.12.13

With great interest, we read the recently published work by van der Leest et al. (1) on European Urology. The authors conducted a multi-reader, prospective study to investigate the diagnostic performance of standard multiparametricMRI (mp-MRI) versus unenhanced biparametric-MRI (bpMRI) in three planes and in one plane ("fast" bp-MRI) to detect high-grade prostatic carcinoma (PCa), in biopsynaïve men. The authors demonstrated that all protocols present a similar diagnostic performance in ruling out highgrade PCa. In particular, a "fast" bp-MRI protocol, only including axial T2w, ADC map, and high b-value images (three image sets), did not result in decreased detection of high-grade PCa. Table 1 summarizes the imaging sequences included in each of the protocols. Even though the negative predictive value (NPV) of "fast" bp-MRI was lower than that of bp-MRI and mp-MRI, the difference in NPV was clinically negligible $(0.15 \%)$, and the NPV of "fast" bp-MRI remained high (97\%). More details regarding diagnostic performance metrics reported using the three different protocols are shown in Table 2. The authors conclude that "fast" unenhanced bp-MRI can double prostate MRI capacity and reduce its costs, without impairing the detection of high-grade PCa.

It is interesting to note that the imaging protocol used by the Authors is not completely in line with current Prostate Imaging - Reporting and Data System (PI-RADS) guidelines. The main issues are the use of a gap in $\mathrm{T} 2 \mathrm{w}$ and diffusion weighted imaging (DWI) sequences and the in-plane resolution of $\mathrm{T} 2 \mathrm{w}$ images. This represents a potential limitation of the study, although reported results and accuracy of PI-RADS scoring does not seem negatively affected. Furthermore, recent publications highlighted the low adherence to PI-RADS acquisition guidelines both in the clinical and academic settings (2-4).

Mp-MRI is considered the technique of choice to evaluate patients with suspicion of PCa, however, the long imaging acquisition time have contributed to a strong interest in reducing the length of mp-MRI. Several papers have demonstrated that bp-MRI protocols represent a valid alternative to mp-MRI (5-7). This is mainly due to the limited added value of dynamic contrast enhanced (DCE) over T2w imaging and DWI when using PI-RADS, for diagnosis of clinically significant PCa $(8,9)$. In this study, van der Leest et al. clearly confirm the high diagnostic accuracy of shortening prostatic MR imaging, in biopsynaïve men with suspicion of PCa. The authors introduce an additional protocol modification and novelty, to further decrease the length of bp-MRI protocols, by eliminating coronal and sagittal $\mathrm{T} 2 \mathrm{w}$ planes, thus reducing the overall acquisition time to 8 minutes. Additional strengths of the manuscript are the multireader evaluation, and a direct cost analysis confirming the benefits in terms of cost reduction (10). This paper nicely demonstrates the value of a "fast" bp-MRI protocol, without the administration of contrast 
Table 1 Included sequences in each of the MRI protocols (1)

\begin{tabular}{lccc}
\hline & MP-MRI & BP-MRI & "FAST" BP-MRI \\
\hline Axial T2w & $\mathbf{0}$ & $\mathbf{\square}$ \\
Sagittal T2w & $\mathbf{0}$ & $\mathbf{\square}$ \\
Coronal T2w & $\mathbf{\square}$ & $\mathbf{\square}$ \\
Axial DWI & $\mathbf{0}$ & $\mathbf{\square}$ \\
3D DCE & $\mathbf{0}$ & \\
\hline
\end{tabular}

BP, biparametric; MP, multiparametric; MRI, magnetic resonance imaging; T2w, T2-weighted sequence; DWI, diffusion weighted imaging sequence; DCE, dynamic contrast enhanced sequence.

Table 2 Reported accuracy values for the three MRI protocols (1)

\begin{tabular}{lllll}
\hline & Sensitivity & Specificity & NPV & PPV \\
\hline MP-MRI & $95(91-97)$ & $69(64-73)$ & $97(94-98)$ & $57(51-62)$ \\
BP-MRI & $95(91-97)$ & $69(64-73)$ & $97(94-98)$ & $57(51-62)$ \\
"FAST" BP-MRI & $95(91-97)$ & $65(61-70)^{\star}$ & $97(94-98)^{\star}$ & $54(49-60)^{\star}$ \\
\hline
\end{tabular}

BP, biparametric; MP, multiparametric; MRI, magnetic resonance imaging; NPV, negative predictive value; PPV, positive predictive value. All values expressed in percentage with $95 \%$ confidence interval in parenthesis. ${ }^{*}$, Statistically significant difference $(\mathrm{P}<0.001)$.

agent, as a feasible tool for PCa detection, significantly reducing both acquisition and interpretation time, while maintaining comparable diagnostic accuracy to bp-MRI and mp-MRI.

Indeed, in recent years evidences in literature have pointed to a greater role for bp-MRI $(11,12)$. As highresolution $\mathrm{T} 2 \mathrm{w}$ images are the most important for lesion morphologic assessment and staging, while DWI and ADC maps for lesion detection and tissue characterization, at least in the peripheral zone, the role of gadolinium-based contrast has become debated (12). Some authors support a significant role in peripheral lesion detection while others advocate its complete removal from routine prostate MRI protocols (11). For example, unenhanced prostate MRI might allow for an accurate assessment of PCa local stage (13). A previous paper underlined that the addition of DCE images does not improve prostate cancer staging accuracy for expert readers (14). This trend is also reflected in the latest revision (v2.1) of the PI-RADS guidelines that acknowledged a role for bp-MRI, even if currently limited to some clinical scenarios (8). For example, mp-MRI should still be employed in patients at high risk for clinically significant PCa.

The future of prostate cancer imaging is not limited to conventional MRI protocols. As in other fields of medicine and especially radiology, there have been a plethora of studies focused on radiomics and machine learning applications (15). These have shown good results in prostate imaging, from gland and lesion segmentation to cancer detection and characterization, as well as tumor staging (16-18). Input data for such analyses has been represented not only by MRI but also from other imaging modalities (19).

In conclusion, the future of prostate MRI looks bright and, just as PI-RADS are an evolving document in accordance to new experiences and data available, so are acquisition protocols. While mp-MRI is still the reference standard, bp-MRI could soon become an equal and strong alternative.

\section{Acknowledgments}

Funding: None.

\section{Footnote}

Conflicts of Interest: All authors have completed the ICMJE uniform disclosure form (available at http://dx.doi. org/10.21037/cco.2019.12.13). The authors have no conflicts of interest to declare.

Etbical Statement: The authors are accountable for all aspects of the work in ensuring that questions related 
to the accuracy or integrity of any part of the work are appropriately investigated and resolved.

Open Access Statement: This is an Open Access article distributed in accordance with the Creative Commons Attribution-NonCommercial-NoDerivs 4.0 International License (CC BY-NC-ND 4.0), which permits the noncommercial replication and distribution of the article with the strict proviso that no changes or edits are made and the original work is properly cited (including links to both the formal publication through the relevant DOI and the license). See: https://creativecommons.org/licenses/by-nc-nd/4.0/.

\section{References}

1. van der Leest M, Israël B, Cornel EB, et al. High Diagnostic Performance of Short Magnetic Resonance Imaging Protocols for Prostate Cancer Detection in Biopsy-naïve Men: The Next Step in Magnetic Resonance Imaging Accessibility. Eur Urol 2019;76:574-81.

2. Cuocolo R, Stanzione A, Ponsiglione A, et al. Prostate MRI technical parameters standardization: A systematic review on adherence to PI-RADSv2 acquisition protocol. Eur J Radiol 2019;120:108662.

3. Burn PR, Freeman SJ, Andreou A, et al. A multicentre assessment of prostate MRI quality and compliance with UK and international standards. Clin Radiol 2019;74:894. e19-894.e25.

4. Esses SJ, Taneja SS, Rosenkrantz AB. Imaging Facilities' Adherence to PI-RADS v2 Minimum Technical Standards for the Performance of Prostate MRI. Acad Radiol 2018;25:188-95.

5. Stanzione A, Imbriaco M, Cocozza S, et al. Biparametric 3T Magentic Resonance Imaging for prostatic cancer detection in a biopsy-naïve patient population: a further improvement of PI-RADS v2? Eur J Radiol 2016;85:2269-74.

6. Kuhl CK, Bruhn R, Krämer N, et al. Abbreviated Biparametric Prostate MR Imaging in Men with Elevated Prostate-specific Antigen. Radiology 2017;285:493-505.

7. Barth BK, De Visschere PJL, Cornelius A, et al. Detection of Clinically Significant Prostate Cancer: Short Dual-Pulse Sequence versus Standard Multiparametric MR Imaging-A Multireader Study. Radiology 2017;284:725-36.

8. Turkbey B, Rosenkrantz AB, Haider MA, et al. Prostate Imaging Reporting and Data System Version 2.1: 2019 Update of Prostate Imaging Reporting and Data System
Version 2. Eur Urol 2019;76:340-51.

9. Cuocolo R, Stanzione A, Rusconi G, et al. PSA-density does not improve bi-parametric prostate MR detection of prostate cancer in a biopsy naïve patient population. Eur J Radiol 2018;104:64-70.

10. Porter KK, King A, Galgano SJ, et al. Financial implications of biparametric prostate MRI. Prostate Cancer Prostatic Dis 2020;23:88-93.

11. Thestrup KCD, Logager V, Baslev I, et al. Biparametric versus multiparametric MRI in the diagnosis of prostate cancer. Acta Radiol Open 2016;5:205846011666304.

12. Junker D, Steinkohl F, Fritz V, et al. Comparison of multiparametric and biparametric MRI of the prostate: are gadolinium-based contrast agents needed for routine examinations? World J Urol 2019;37:691-9.

13. Stanzione A, Ponsiglione A, Cuocolo R, et al. Abbreviated Protocols versus Multiparametric MRI for Assessment of Extraprostatic Extension in Prostatic Carcinoma: A Multireader Study. Anticancer Res 2019;39:4449-54.

14. Fütterer JJ, Engelbrecht MR, Huisman HJ, et al. Staging Prostate Cancer with Dynamic Contrast-enhanced Endorectal MR Imaging prior to Radical Prostatectomy: Experienced versus Less Experienced Readers. Radiology 2005;237:541-9.

15. Gillies RJ, Kinahan PE, Hricak H. Radiomics: Images Are More than Pictures, They Are Data. Radiology 2016;278:563-77.

16. Cuocolo R, Cipullo MB, Stanzione A, et al. Machine learning applications in prostate cancer magnetic resonance imaging. Eur Radiol Exp 2019;3:35.

17. Bonekamp D, Kohl S, Wiesenfarth M, et al. Radiomic Machine Learning for Characterization of Prostate Lesions with MRI: Comparison to ADC Values. Radiology 2018;289:128-37.

18. Cuocolo R, Stanzione A, Ponsiglione A, et al. Clinically significant prostate cancer detection on MRI: A radiomic shape features study. Eur J Radiol 2019;116:144-9.

19. Cytawa W, Seitz AK, Kircher S, et al. 68Ga-PSMA I\&T PET/CT for primary staging of prostate cancer. Eur $\mathbf{J}$ Nucl Med Mol Imaging 2020;47:168-77.

Cite this article as: Imbriaco M, Stanzione A, Tamburi V, Gambardella M, Ponsiglione A, Cuocolo R. Are short magnetic resonance imaging protocols the future of prostate imaging? Chin Clin Oncol 2020;9(3):41. doi: 10.21037/cco.2019.12.13 